\title{
The development of truncated inviscid turbulence and the Fermi-Pasta-Ulam problem
}

\author{
G. Ooms and B. J. Boersma \\ J. M. Burgerscentrum, Delft University of Technology, Faculty of Mechanical Engineering, \\ Laboratory for Aero- and Hydrodynamics, Mekelweg 2, 2628 CD Delft, The Netherlands
}

(Received 18 August 2008; accepted 21 November 2008; published online 18 December 2008)

\begin{abstract}
A study was made of the possible similarity between the development of truncated, inviscid turbulence and the Fermi-Pasta-Ulam (FPU) problem. For the case of a constant time scale, which resembles the FPU problem, a significant increase in the time to achieve equipartition was found when the initial energy was decreased. At first a few modes were generated and only rather late in the spectral development other modes appear and equipartition was established. (C) 2008 American Institute of Physics. [DOI: 10.1063/1.3046581]
\end{abstract}

As is well known Fermi, Pasta, and Ulam ${ }^{1}$ (FPU) studied the energy redistribution between the linear modes of a one-dimensional chain of particles connected via weakly nonlinear springs. To their surprise no apparent tendency to equipartition of energy was observed in their numerical experiments. Much more knowledge is now available about this problem (see, for instance, the recent book by Gallavotti ${ }^{10}$ or the review by Campbell et al. ${ }^{9}$ in the Focus Issue on the FPU problem in the journal Chaos). According to more recent studies an exponential localization of the energy distribution in normal mode space is observed on a very short time scale $\tau_{1}$. It possesses a core with a few modes that are strongly excited, and a tail of exponentially weakly excited modes. Below an energy threshold there is a slow pumping of energy from the core of the distribution into its tail. This process ultimately brings the system to equipartition, but on a much larger time scale $\tau_{2}\left(\tau_{2} \gg \tau_{1}\right)$. In this paper we present the results of an investigation of another system of nonlinearly coupled normal modes to find out whether results similar to the FPU results are found. This system is truncated, inviscid turbulence. To that purpose we carried out direct numerical simulations of the turbulent flow of an inviscid fluid inside a 3D box. Only a limited number of modes was taken into account. The purpose of our calculations was to start with energy in one normal mode and then study the development of the energy spectrum as a function of time with special interest for the influence of the amount of initial energy on the development, to see whether results were found similar to the FPU problem. As with the FPU problem we found that also for inviscid turbulence the equipartition of energy over the entire normal mode spectrum is strongly slowed down when the turbulent kinetic energy decreases. FPU observed recurrences during the development of the spectrum of almost all the energy into the initially excited mode. Such surprising recurrences were not observed by us for the turbulence problem. However, we found that first a few modes were generated and only rather late in the spectral development other modes appear and equipartition was established. Although the FPU problem deals with a different physical problem (dynamics of a 1D chain of par- ticles connected via springs) than our problem (dynamics of an inviscid fluid flow inside a 3D box), the influence of a decrease in the energy of the system on the delay in the development of the energy spectrum is rather similar for both problems.

\section{INTRODUCTION}

In 1953 Fermi, Pasta, and Ulam (FPU) (Ref. 1) studied the problem of energy redistribution between the linear normal modes of a one-dimensional chain of particles with nearest neighbor interactions (see Fig. 1). They assumed that there are $N$ particles. The leftmost particle $(n=1)$ is connected via a spring to the left wall, the rightmost particle $(n=N)$ via a spring to the right wall and the particles are mutually connected via springs. The springs have a linear and a weakly nonlinear component described by the following potential:

$$
V(r)=\frac{1}{2} r^{2}+\frac{\alpha}{3} r^{3}+\frac{\beta}{4} r^{4},
$$

in which $r$ is the distance between the particles and $\alpha$ and $\beta$ are constants.

In their numerical calculations FPU solved the following equation of motion for the particles in case of the $\alpha$-model $(\beta=0)$ :

$$
\ddot{x}_{n}=\left(x_{n+1}-2 x_{n}+x_{n-1}\right)+\alpha\left[\left(x_{n+1}-x_{n}\right)^{2}-\left(x_{n}-x_{n-1}\right)^{2}\right]
$$

or the following equation of motion in case of the $\beta$-model $(\alpha=0)$ :

$$
\ddot{x}_{n}=\left(x_{n+1}-2 x_{n}+x_{n-1}\right)+\beta\left[\left(x_{n+1}-x_{n}\right)^{3}-\left(x_{n}-x_{n-1}\right)^{3}\right] .
$$

The coordinate $x_{n}(t)$ represents the displacement of the $n$th particle from its equilibrium position. All particles have unit mass and the elastic spring constant has also been taken equal to unity. At the two walls fixed boundary conditions were used, so $x_{0}=0$ and $x_{n+1}=0$. In the absence of nonlinear terms in Eqs. (2) and (3) the normal modes of the possible 


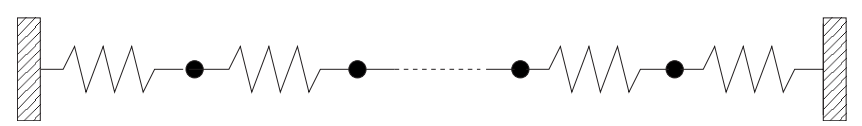

FIG. 1. Sketch of chain of $N$ particles connected via springs.

movements of the chain can be expressed as the following Fourier representation of the displacements:

$$
x_{n}(t)=\left(\frac{2}{N+1}\right)^{1 / 2} \sum_{k=1}^{N} Q_{k}(t) \sin \left(\frac{\pi k n}{N+1}\right),
$$

in which $Q_{k}(t)$ is the normal mode amplitude. The mode number $k=1, \ldots, N$ relates each of these modes to its corresponding normal mode frequency $\omega_{k}=2 \sin [\pi k / 2(N+1)]$.

FPU gave the particles (with $N=32$ ) an initial displacement according to a fundamental mode of the linear system, for instance, $k=1$, and the equation of motion (2) or (3) was integrated on their computer. They expected that after some time other modes would become excited as well, and that the energy would eventually be equally distributed over the entire spectrum. The reason is that according to the ergodic hypothesis the dynamical trajectory of the system in phase space (given sufficient time) will visit all microstates consistent with the applied constraints. However, to their surprise no apparent tendency to equipartition of energy was observed in their numerical experiment. They found instead that, up to the maximum computing time available (which was orders of magnitude larger than the largest periods of oscillation of the normal modes) the energy was shared only within a small packet of low-frequency modes, with no approach to equipartition at all. The time-average energy spectrum seemed to approach an equilibrium, but this equilibrium spectrum had nothing to do with equipartition of energy. It showed an exponential decay towards the high frequencies. Recurrences of almost all the energy into the initially excited mode were observed as well. Many later studies showed that these results could not be trivialized by accusing the low accuracy of the integration scheme employed by FPU.

From later studies it became clear that if one repeated the calculations for initial data of the same type as used by FPU, but with large enough energy in the initially excited mode or with a large enough number of initially excited modes, the spectrum was found to relax within the available time to the flat spectrum corresponding to equipartition. So it appeared that there exists an energy threshold and system size threshold above which equipartition is observed on a relatively short time scale. According to Flach et al. ${ }^{2}$ the situation can be described in the following way. An exponential localization of the energy distribution in normal mode space is observed on a very short time scale $\tau_{1}$. It possesses a core with a few modes that are strongly excited, and a tail of exponentially weakly excited modes. Below the energy threshold or system size threshold there is a slow pumping of energy from the core of the distribution into its tail. This process ultimately brings the system to equipartition, but on a much larger time scale $\tau_{2}\left(\tau_{2} \gg \tau_{1}\right)$. If the initial energy is increased, the energy threshold (which depends on the system size) may be reached for which the time scale of the core-tail pumping becomes comparable with the time needed for the energy transition to the exponential distribution $\left(\tau_{2}\right.$ $\sim \tau_{1}$ ). The same observation holds for the system size threshold (which depends on the energy threshold). Below these threshold values the time scales $\tau_{2}$ and $\tau_{1}$ differ by orders of magnitude. By chance, FPU chose their initial condition in their numerical experiment below the energy threshold. With a stronger initial excitation no FPU problem would have arisen, because equipartition of energy would have been observed even with the relatively short integration time that the authors could afford in 1953.

We have the following remark concerning the FPU problem. We start from the equation of motion of one of the particles in the chain

$$
M \frac{d v}{d t}=\left(C r+\alpha r^{2}\right)_{r}-\left(C r+\alpha r^{2}\right)_{l},
$$

in which $M$ is the mass of the particle, $v$ its velocity, $t$ the time, and $C$ the spring constant. The index $r$ refers to the interaction of the particle with its neighbor to the right, the index $l$ refers to the interaction with its neighbor to the left. We make Eq. (5) dimensionless by means of the velocity scale $V_{0}=E_{0}^{1 / 2}$, the time scale $T_{0}$, and the length scale $L_{0}$ $=T_{0} V_{0}=T_{0} E_{0}^{1 / 2} \cdot E_{0}$ is the energy of the particle at $t=0$ due to its initial displacement. The resulting dimensionless equation is

$$
\begin{aligned}
\frac{d v^{\star}}{d t^{\star}}= & {\left[\left(\frac{C T_{0}^{2}}{M}\right) r^{\star}\right]_{r}-\left[\left(\frac{C T_{0}^{2}}{M}\right) r^{\star}\right]_{l}+\left[\left(\frac{E_{0}^{1 / 2} \alpha T_{0}^{3}}{M}\right) r^{\star 2}\right]_{r} } \\
& -\left[\left(\frac{E_{0}^{1 / 2} \alpha T_{0}^{3}}{M}\right) r^{\star 2}\right]_{l}
\end{aligned}
$$

where $v^{\star}=v / V_{0}, t^{\star}=t / T_{0}$, and $l^{\star}=l / L_{0}$. As already mentioned, FPU started their calculation with an initial displacement according to a fundamental mode of the linear system, for instance, $k=1$. The frequency of that mode is given by $\omega=2 \sin [\pi / 2(N+1)]$ and we choose as a representative time scale $T_{0}=2 \pi / \omega$. Equation (6) gives us some insight, as to what happened during the FPU calculations when the initial energy $E_{0}$ was reduced. As $\left[\left(C T_{0}^{2} / M\right)\right]_{r}$ and $\left[\left(C T_{0}^{2} / M\right)\right]_{l}$ do not depend on the energy $E_{0}$ of the initial displacement, the two terms $\left[\left(E_{0}^{1 / 2} \alpha T_{0}^{3} / M\right)\right]_{r}$ and $\left[\left(E_{0}^{1 / 2} \alpha T_{0}^{3} / M\right)\right]_{l}$ became increasingly smaller with decreasing $E_{0}$ than the other terms in Eq. (6). As these two terms describe the nonlinear interaction between the modes of the system, this means that with decreasing $E_{0}$ the pumping of energy from the first excited mode into other modes slowed down, as found by the FPU calculations.

Another remark concerning the FPU problem is the fact that, while the length scale of the particle movement decreases with decreasing initial energy as $L_{0}=T_{0} E_{0}^{1 / 2}$, the length scale of the total system, i.e., the distance between the walls, remains constant.

However, the aim of our study is not to study a new aspect of the FPU problem. Much knowledge is already available about it (see, for instance, Refs. 3-8, and the review by Campbell et al. ${ }^{9}$ in the Focus Issue on the FPU problem in the journal Chaos). There is also a recent book by Gallavotti. ${ }^{10}$ In this paper we present the results of an inves- 
tigation of another system of nonlinearly coupled normal modes to find out whether results similar to the FPU results are found. This system is truncated, inviscid turbulence.

\section{DEVELOPMENT OF TRUNCATED, INVISCID TURBULENCE}

\section{A. Calculation procedure}

Consider a fluid inside a 3D box. The length of the sides is given by $L=2 \pi$. The fluid is assumed to be inviscid and incompressible. By stirring it for a certain time it is put into a turbulent motion. Because the fluid is inviscid, the kinetic energy of the flow field will not change after the stirring is stopped. The equation of motion describing the flow inside the box is given by

$$
\frac{\partial \mathbf{v}}{\partial t}+(\mathbf{v} \cdot \nabla) \mathbf{v}=-\frac{1}{\rho_{f}} \nabla p,
$$

in which $\mathbf{v}$ is the fluid velocity, $p$ the pressure, $\rho_{f}$ the fluid density, and $t$ is time. We assume periodic boundary conditions at the walls of the box. If the nonlinear term is omitted, the equation of motion becomes

$$
\frac{\partial \mathbf{v}}{\partial t}=-\frac{1}{\rho_{f}} \nabla p .
$$

Without the nonlinear term, the flow field can be decomposed in independent normal modes

$$
\mathbf{v}(\mathbf{x}, t)=\sum_{\mathbf{k}} \mathbf{v}(\mathbf{k}, t)[\exp (-\mathbf{k} \cdot \mathbf{x})],
$$

with $\mathbf{v}(\mathbf{k}, t)$ the amplitude belonging to the normal mode with wave number $\mathbf{k}$. ( $\mathbf{x}$ is the three-dimensional space vector with components $x_{1}, x_{2}$, and $x_{3}$.) $\mathbf{k}$ has components $k_{1}, k_{2}$, and $k_{3}$ that can each have the value $n 2 \pi / L$ with $n$ running from 1 to $\infty$. However, in our calculations we can take into account only a limited number of modes.

Equation (7) is solved by means of direct numerical simulation (DNS). The velocity and pressure field is represented as a truncated Fourier series. Spatial derivatives are calculated in Fourier space. The nonlinear term has been evaluated in physical space. The time integration was performed with a standard second-order Adams-Basforth method. This is a well-known scheme for fluid flow simulations. A good reference book on the Adams-Basforth method is the book by Gear. ${ }^{11}$

We will study in particular the one-dimensional energy spectrum $E_{1}\left(k_{1}, t\right)=\frac{1}{2}\left[v_{1}\left(k_{1}, t\right)\right]^{2}$, in which $v_{1}(k, t)$ is the $\mathbf{v}$-component in the $x_{1}$-direction. This is done in the following way. At a certain time step and for a certain combination of values for $x_{2}$ and $x_{3}$ the energy spectrum $E_{1}\left(k_{1}, t\right)$ is calculated from the $v_{1}$-values at the grid points along the $x_{1}$-line. At that same time step this calculation is repeated for all other combinations of $\left(x_{2}, x_{3}\right)$-values and the results are summed for each value of $k_{1}$ yielding the $E_{1}\left(k_{1}\right)$-spectrum at the chosen time step. This calculation is repeated at every time step, so the final result is the energy spectrum as function of time $E_{1}\left(k_{1}, t\right)$. As only a limited number of grid points are taken into account the spectrum is truncated. In the same way also the spectra $E_{2}\left(k_{2}, t\right)$ and $E_{3}\left(k_{3}, t\right)$ can be deter- mined. In case the flow field is isotropic the three spectra are the same. In our calculation we will start with a nonisotropic flow field and so the spectra will be different. When a fully developed turbulent flow field develops in time an isotropic state can be reached. We are, however, in particular interested in the development of the initial state, which is not isotropic. As there is no energy loss, the total energy (which is equal to the integral over the spectrum) remains constant and is the same for the three spectra $E_{1}\left(k_{1}, t\right), E_{2}\left(k_{2}, t\right)$, and $E_{3}\left(k_{3}, t\right)$.

The purpose of our calculations is to start with energy in one normal mode and then study the development of the energy spectrum $E_{1}$ as a function of time with special interest for the influence of the amount of initial energy on the development, to see whether results are found similar to the FPU problem. In our calculations we used 96 grid points in each direction of $\mathbf{x}$-space, so the number of modes that were calculated in each direction in $k$-space was 48 . At each time step we kept the results for the 24 modes with the smallest wave numbers. The energy of the 24 modes with the largest wave numbers was put equal to zero after each time step. This was done for the following reason. Due to the nonlinear interaction between the modes, energy can be transferred over the truncation surface in $\mathbf{k}$-space between the modes with the smallest wave numbers and those with the largest ones and we wanted to investigate how important this effect is. We found no energy transfer over the truncation surface, as long as the energy is only present in a few modes with small wave numbers. However, during the calculation the energy spreads gradually to modes with larger wave numbers and finally reaches the truncation surface. From that moment an energy transfer from the 24 modes with the smallest wave numbers to the 24 modes with the largest wave numbers occurs. This energy loss (per unit time) increases with increasing time step in the numerical calculations. The energy loss as function of time starts at a very low level, but increases strongly with time. So in order to avoid the energy loss in the 24 smallest-wave-number modes, we kept the time step in the DNS calculations small. We always checked during the calculation that the total energy present in the $E_{1}$-spectrum (or the other spectra) remained constant. So all the energy was present in the 24 smallest-wave-number modes; in the 24 largest-wave number modes the energy is kept zero.

\section{B. Comparison with theoretical results}

We first compare our DNS-calculations against known theoretical results. We first check whether the spectrum does not change as function of time, in case the nonlinear term is absent. We choose an initial flow field that has decreasing energy with increasing wave number and determine the development in time of the flow field by means of a DNS calculation.

The initial flow field is arbitrary, although it is chosen in such a way that there is no helicity $\omega \cdot \mathbf{v} . \omega$ is the vorticity of the flow field. We will come back to this point later on. The results of our calculation in terms of the energy spectrum are given in Fig. 2. The figure shows the spectrum $E_{1}$ as a func- 


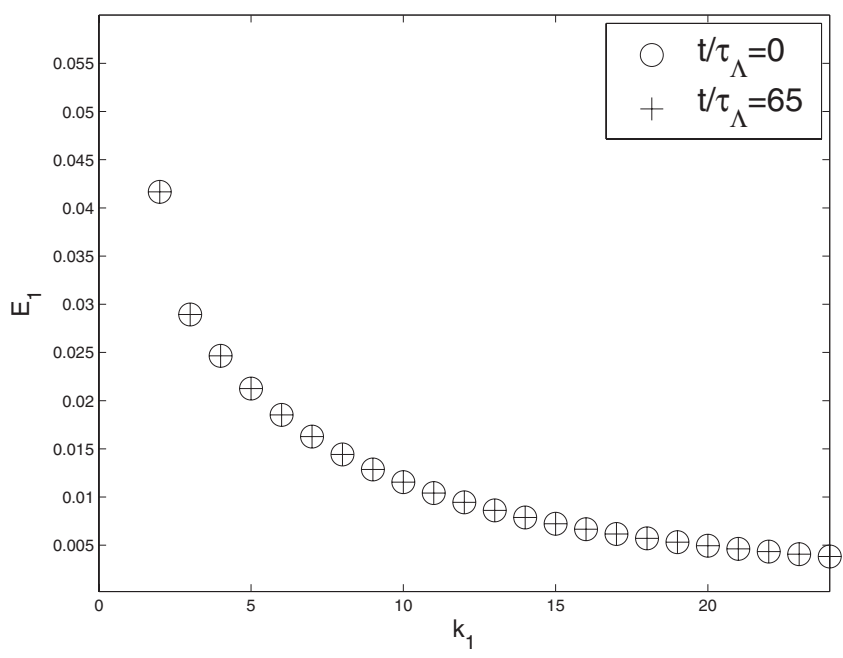

FIG. 2. Spectrum $E_{1}$ as a function of wave number $k_{1}$ at two values of $t / \tau_{\Lambda}$ for the case where there is no nonlinear term in the equation of motion. As can be seen the spectrum does not change.

tion of wave number $k_{1}$ at two different times $t / \tau_{\Lambda}=0$ and $t / \tau_{\Lambda}=65$. $\tau_{\Lambda}$ is the time scale of the normal mode with the largest energy (and largest length scale). It is found by dividing the length scale of that mode by its velocity scale. The length scale is found by dividing the length scale of the flow domain $(2 \pi)$ by the wave number of the mode. The velocity scale is derived by taking the square root of the energy of the mode. As can be seen from Fig. 2, even after $65 \tau_{\Lambda}$, the spectrum has not changed. This is, of course, due to the absence of interaction between the modes. Also the flow field has not changed.

Next we repeat this calculation, but this time with the nonlinear term. The result for $E_{1}$ is shown in Fig. 3. Equipartition of energy over the normal modes has taken place within one time scale $\tau_{\Lambda}$. This is also well-known from "ordinary" turbulence theory with viscous dissipation: the cascade process due to the nonlinear interaction between the modes transfers most of the energy within the integral time

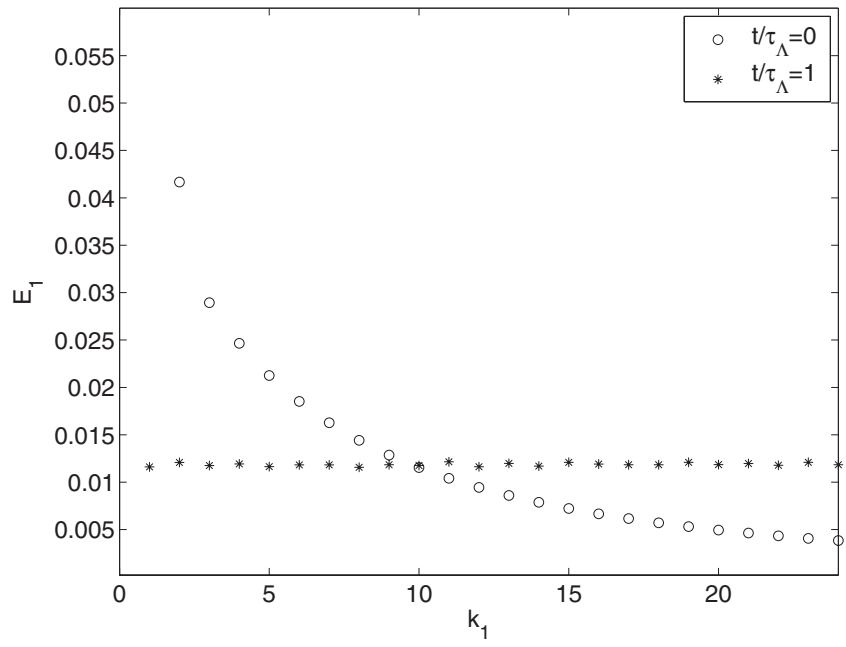

FIG. 3. Spectrum $E_{1}$ as a function of wave number $k_{1}$ at two values of $t / \tau_{\Lambda}$ for the case that the nonlinear term is included in the equation of motion. As can be seen there is equipartition of energy over the normal modes within one time scale $\tau_{\Lambda}$.

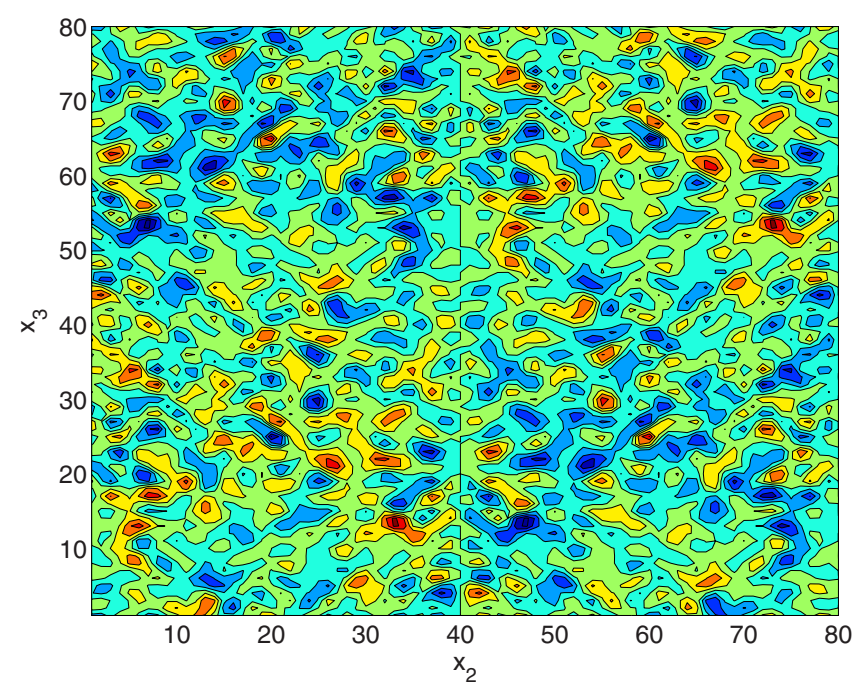

FIG. 4. (Color online) Cross section of the $v_{1}$-component of the flow field after $t / \tau_{\Lambda}=1.3$.

scale from the largest modes to the smallest ones where the turbulence energy is dissipated (see, for instance, Taylor ${ }^{12}$ ). The cross section of the $v_{1}$-component of the flow field at $t / \tau_{\Lambda}=1.3$ is given in Fig. 4 .

We also calculated the development of an initial flow field that has increasing energy with increasing wave number (see Fig. 5, in which again $\tau_{\Lambda}$ is the time scale of the mode with the smallest wave number). As can be seen the development yields also in this case an equilibrium spectrum with equipartition of energy over all the normal modes. So in wave number space the energy transfer goes in both directions: from the small wave numbers to the large ones and vice versa.

There are two constants of motion for a turbulent inviscid flow field: the total energy and the total helicity of the flow field. All results presented so far were for the case that the helicity is zero and so the total energy is then the only constant of motion. According to Kraichnan, ${ }^{13}$ the equilib-

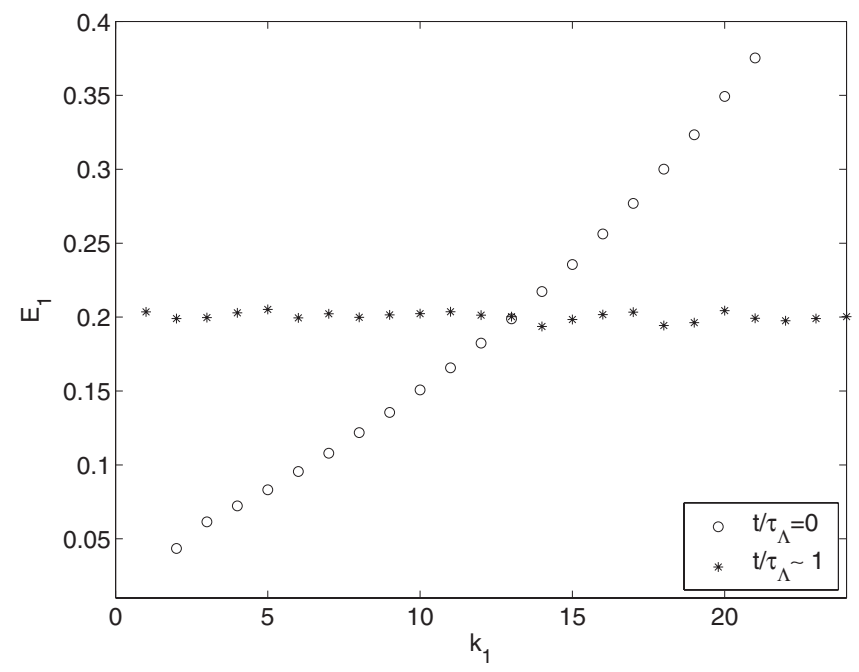

FIG. 5. Spectrum $E_{1}$ as a function of wave number $k_{1}$ at two values of $t / \tau_{\Lambda}$ for the case of an initial flow field that has increasing energy with increasing wave number. 


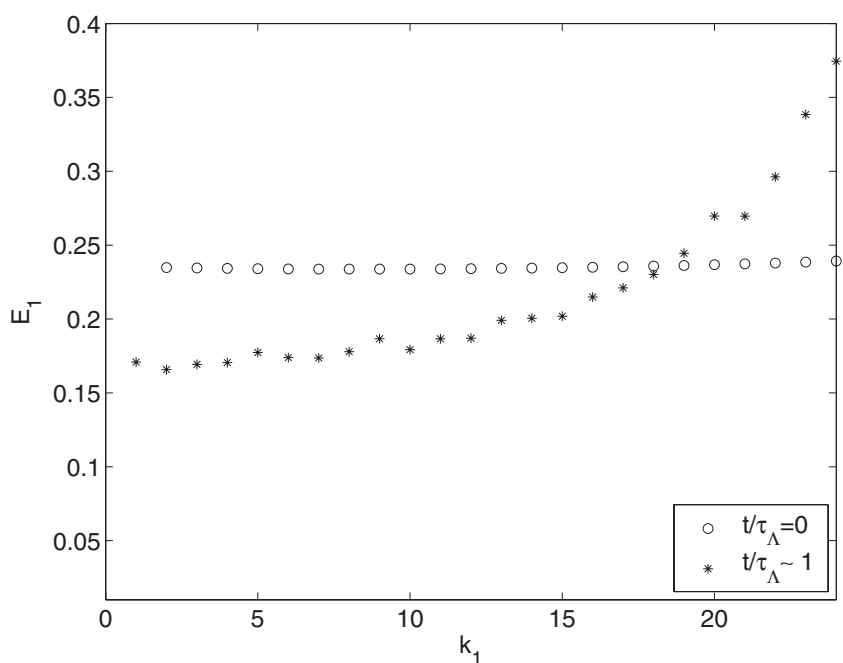

FIG. 6. Spectrum $E_{1}$ as a function of wave number $k_{1}$ at two values of $t / \tau_{\Lambda}$ for the case of a turbulent flow field with maximum helicity.

rium energy spectrum in the case of maximum helicity is not equipartition of energy over all the normal modes, but this spectrum is given by $E(k)=2 \gamma /\left(\gamma^{2}-\delta^{2} k^{2}\right)$. The parameters $\gamma$ and $\delta$ are constrained by $\gamma>0$ and $\delta k<\gamma$. To check this statement by Kraichnan we generated an initial flow field with maximum helicity (using a method described by Polifke ${ }^{14}$ ) and with an almost uniform spectral energy distribution, and calculated its development in time. The result is shown in Fig. 6 and indeed the spectrum takes the form as calculated analytically by Kraichnan. The values of $\gamma$ and $\delta$ for this case can be found by fitting the theoretical spectrum $E(k)=2 \gamma /\left(\gamma^{2}-\delta^{2} k^{2}\right)$ to the values of the calculated points. The time needed to achieve this new form of equilibrium spectrum is again about $\tau_{\Lambda}$ (the time scale of the mode with the smallest wave number). All further results in this paper are for the case that the helicity is zero and so the total energy is then the only constant of motion.

\section{Influence of decreasing initial energy (constant length scale)}

After checking our calculations against known theoretical results we are now in a position to study the influence of decreasing initial energy on the energy transfer between the modes and compare the results with those for the FPU problem. We choose an initial spectrum consisting of one excited normal mode and study the development of its spectrum in time.

Before doing that we study the equation of motion (7) in dimensionless form. As with the equation of motion for the chain we choose for the velocity scale $E_{0}^{1 / 2}$. As length scale the size of the flow field belonging to the excited mode $L_{0}$ is chosen. So the time scale is given by $T_{0}=L_{0} V_{0}=L_{0} / E_{0}^{1 / 2}$. With $v^{\star}=v / V_{0}, t^{\star}=t / T_{0}$, and $l^{\star}=l / L_{0}$ this leads to the following dimensionless equation of motion for the flow field:

$$
\frac{\partial \mathbf{v}^{\star}}{\partial t^{\star}}+\left(\mathbf{v}^{\star} \cdot \nabla^{\star}\right) \mathbf{v}^{\star}=-\nabla^{\star} p^{\star} \text {. }
$$

There is an essential difference with Eq. (6). Equation (10) does not change, when the initial energy is changed. So the

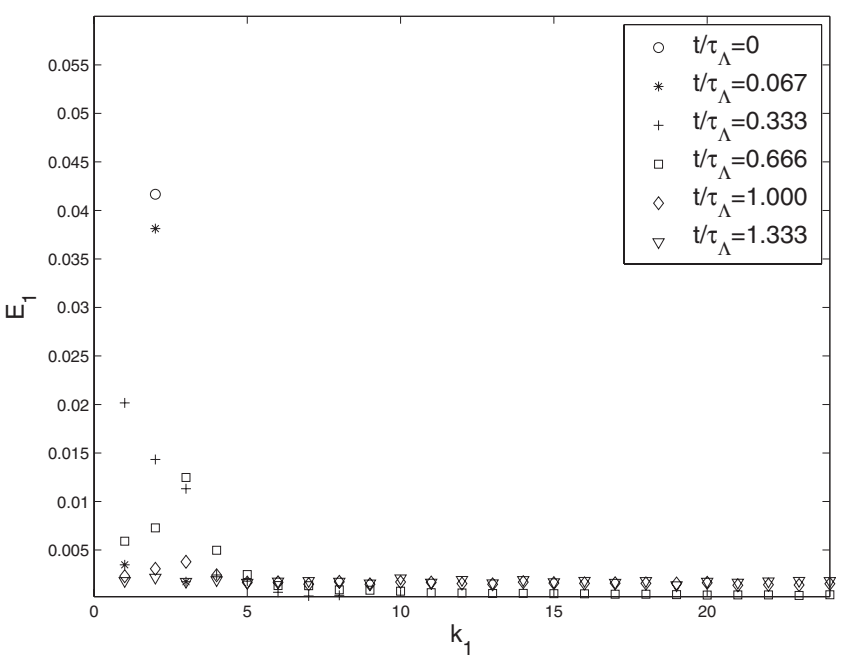

FIG. 7. Spectrum $E_{1}$ as a function of wave number $k_{1}$ at six values of $t / \tau_{\Lambda}$ for the case of one excited initial mode.

results for different values of $E_{0}$ can be scaled according to the dimensionless variables given above. To check this statement we have carried out two DNS calculation for the spectral development of the initial excited mode (mentioned above) with a difference in initial energy of a factor 100 . The results are given in Figs. 7 and 8 , in which $\tau_{\Lambda}$ and $\tau_{\Lambda, 1 \%}$ are the integral time scales for the two cases, respectively.

Indeed, as expected the energy distribution is the same for the two cases when the energy is divided by a factor of $10^{-2}$ and the time multiplied by a factor of $10\left(\tau_{\Lambda, 1 \%}=10 \tau_{\Lambda}\right.$ as the length scale remains the same and the velocity scale is ten times smaller for the $1 \%$ case).

As can be seen from Figs. 7 and 8 after about one integral time scale (respectively, $\tau_{\Lambda}$ and $\tau_{\Lambda, 1 \%}$ ) equipartition of energy over all the modes has taken place for both cases. As $\tau_{\Lambda, 1 \%}=10 \tau_{\Lambda}$ the influence of the amount of initial energy on the energy transfer process between the modes is significant. At an integral time scale $\tau_{\Lambda} \sim 1$ equipartition of energy is

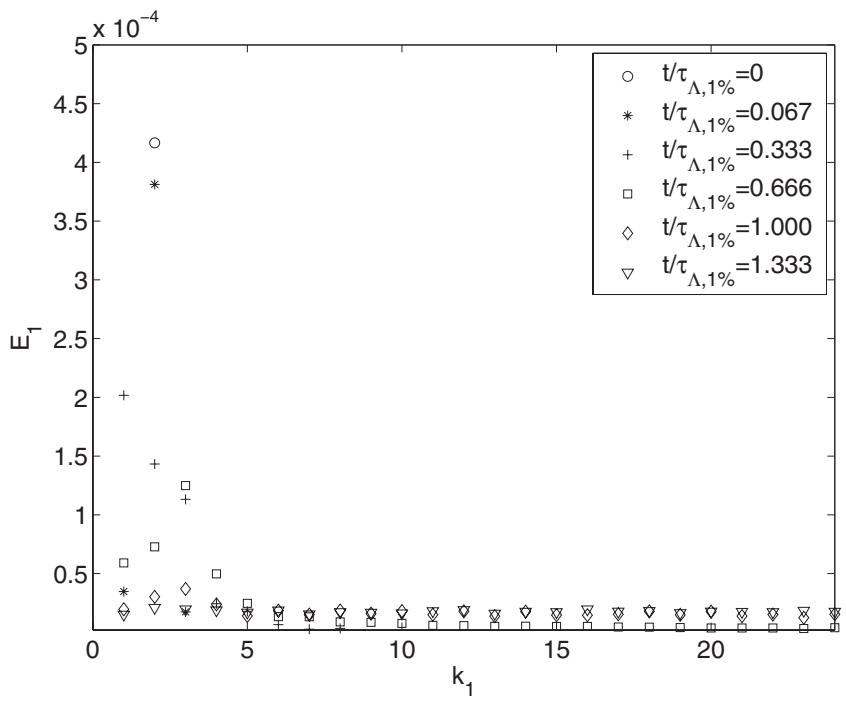

FIG. 8. Spectrum $E_{1}$ as a function of wave number $k_{1}$ at six values of $t / \tau_{\Lambda, 1 \%}$ for the case of one excited initial mode. The initial mode is the same as in Fig. 7 but with $1 \%$ of the energy of that mode. 


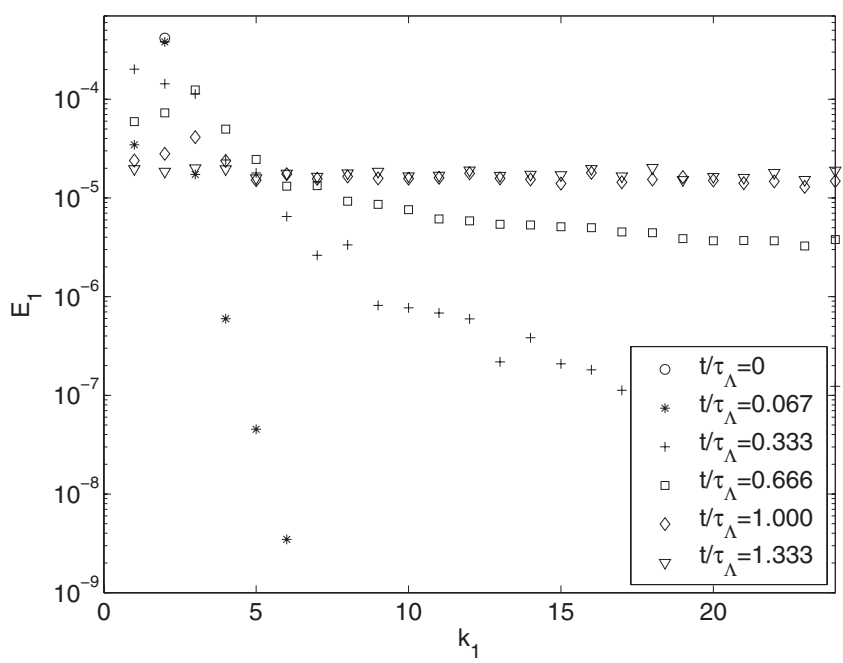

FIG. 9. Spectrum $E_{1}$ as a function of wave number $k_{1}$ as shown in Fig. 8, but now with a log scale for the energy axis. As can be seen, the spectrum possesses a core with a few modes that are strongly excited, and a tail of exponentially weakly excited modes.

still absent for the case of Fig. 8. Only after about $\tau_{\Lambda} \sim 10$ has equipartition been achieved. For the case of Fig. 7 equipartition already occurs at $\tau_{\Lambda} \sim 1$. However these results are rather trivial, as they can be explained on the basis of the scaling rules for the dimensionless variables of Eq. (10).

In Fig. 9 the spectrum shown in Fig. 8 is plotted with a $\log$ scale for the energy axis. As can be seen the spectrum possesses a core with a few modes that are strongly excited, and a tail of exponentially weakly excited modes. There is a slow pumping of energy from the core of the distribution into its tail, similar to the result of Flach et al. ${ }^{2}$

\section{Influence of decreasing initial energy (constant time scale)}

We will now study the influence of decreasing initial energy while keeping the time scale $T_{0}$ constant. As velocity scale is again chosen $V_{0}=E_{0}^{1 / 2}$. So the length scale is given by $L_{0}=V_{0} T_{0}=E_{0}^{1 / 2} T_{0}$. This means that the length scale decreases with decreasing initial energy. When we would decrease in the same way, the length scale of the total system (consisting of all the flow structures), the scaling rules discussed in the last paragraph would hold again for this case. However, we keep the length scale of the total system constant. As mentioned earlier this was also the case for the FPU problem, i.e., the distance between the two walls remained the same when the amplitude of the excitation was changed. The aim of our study is to simulate as much as possible for truncated inviscid turbulence the conditions for the FPU problem and then investigate the possible similarities.

We start again with the calculation of the spectral development for an initial spectrum with one excited mode. The result already given in Fig. 7 is repeated in Fig. 10. Then we decrease the initial energy $E_{0}$ by a factor of 4 , while decreasing the length scale $L_{0}$ of the initial mode by a factor of 2 and calculate again the spectral development. We repeat such calculation, but this time by decreasing the initial energy by a factor of 25 and the length scale of the initial mode by a

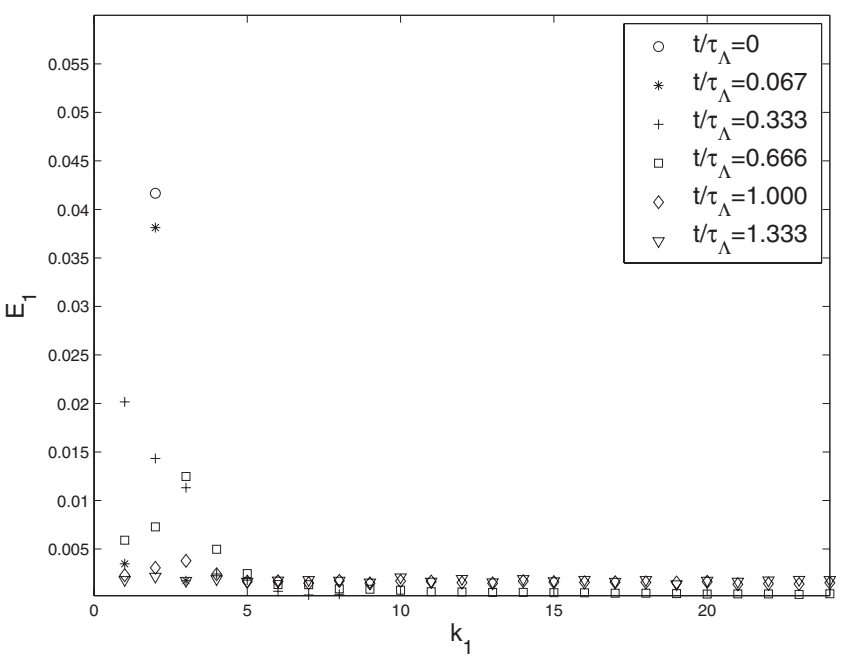

FIG. 10. Spectrum $E_{1}$ as a function of wave number $k_{1}$ at six values of $t / \tau_{\Lambda}$ for the case of one excited initial mode.

factor of 5. The results are, respectively, given in Figs. 11 and 12, in which $\tau_{\Lambda}$ is the integral time scale for the case shown in Fig. 10. It can be seen from the figures that with decreasing energy the equipartition of energy is increasingly delayed. For a decrease in energy of a factor 4 the equipartition takes about a factor 3 more time than for the base case shown in Fig. 10 and for a decrease in energy of a factor of 25 equipartition is already delayed by a factor of 10 . At first a few modes are generated and the change in energy of these modes continues for a relatively long time. Then the remaining modes suddenly appear and equipartition of energy is rather quickly established. This can even be better seen in Fig. 13, in which the results of Fig. 12 are plotted with a log scale for the energy axis. The spectrum possesses a few modes that are strongly excited, but a tail of exponentially weakly excited modes is not observed. Contrary to the result shown in Fig. 9 there is a rather flat spectrum of very weakly

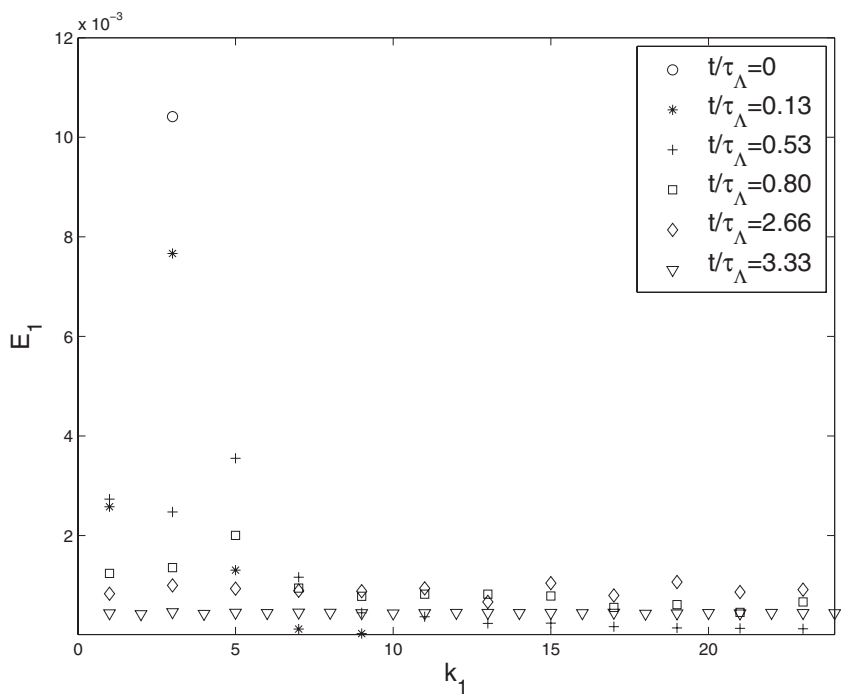

FIG. 11. Spectrum $E_{1}$ as a function of wave number $k_{1}$ at six values of $t / \tau_{\Lambda}$ for the case of one excited initial mode. The initial energy is a factor 4 smaller than for the case shown in Fig. 10 and the length scale of the initial mode is a factor of 2 smaller. 


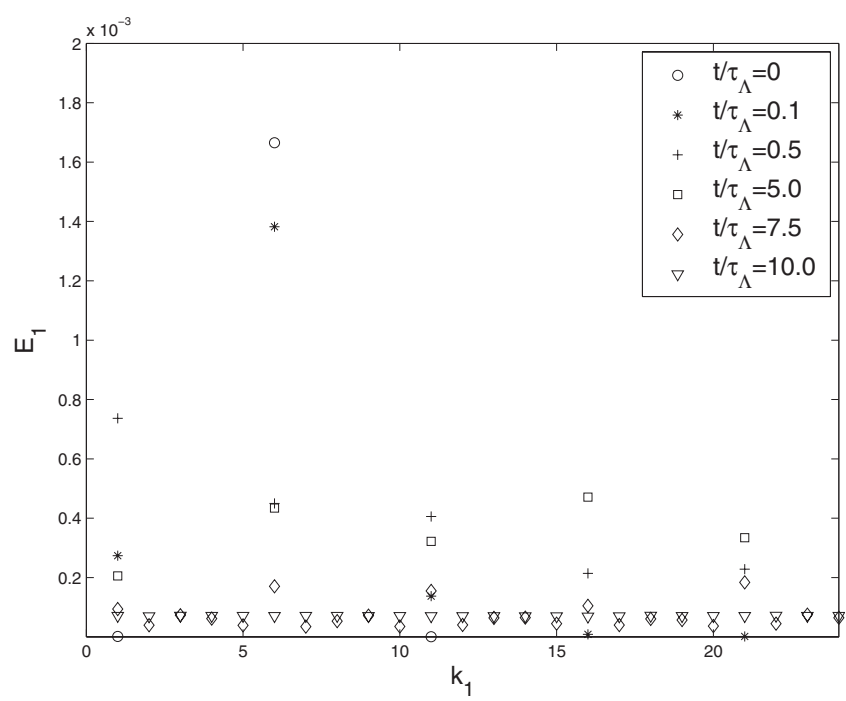

FIG. 12. Spectrum $E_{1}$ as a function of wave number $k_{1}$ at six values of $t / \tau_{\Lambda}$ for the case of one excited initial mode. The initial energy is a factor 25 smaller than for the case shown in Fig. 10 and the length scale of the initial mode is a factor of 5 smaller.

excited modes. Only rather late in the spectral development they increase in strength and equipartition is established.

For the results shown in Figs. 11 and 12 we have made an estimate of the time scale $\tau_{1}$ for the initial localization of the energy distribution with a few excited modes in normal mode space and for the time scale $\tau_{2}$ for reaching equipartition. For Fig. 11 the ratio $\tau_{2} / \tau_{1}$ is approximately equal to 15 , whereas for Fig. 12 the ratio $\tau_{2} / \tau_{1}$ is approximately 50. As the time scale $\tau_{1}$ is nearly the same for the two cases, this means that the time scale $\tau_{2}$ for reaching equipartition is strongly increasing with decreasing initial energy. This is in qualitative accordance with the results for the FPU problem.

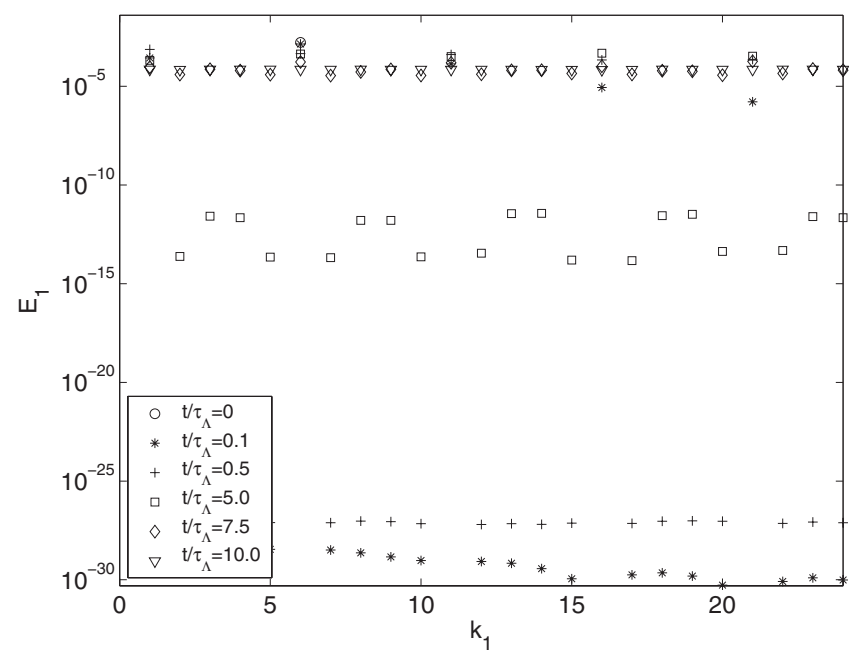

FIG. 13. Spectrum $E_{1}$ as a function of wave number $k_{1}$ as shown in Fig. 12, but now with a log scale for the energy axis. As can be seen the spectrum possesses a few modes that are strongly excited, but a tail of exponentially weakly excited modes is not observed. There is a rather flat spectrum of weakly excited modes. Only rather late in the spectral development they increase in strength and equipartition is established.

\section{DISCUSSION}

As with the FPU problem we found that also for inviscid turbulence, the equipartition of energy over the entire normal mode spectrum is strongly slowed down when the turbulent kinetic energy decreases. For the case of a constant length scale this can be explained by the growth of the characteristic time scale of turbulence with decreasing turbulent energy. For the case of a constant time scale, such explanation is no longer possible. This case, which is more similar to the FPU problem, also shows a very significant increase in the time to achieve equipartition with decreasing initial energy. For this case first a few modes were generated and only rather late in the spectral development other modes appear and equipartition was established.

FPU observed recurrences during the development of the spectrum of almost all the energy into the initially excited mode. Such surprising recurrences were not observed by us for the turbulence problem. However, recurrence is not a universal feature of the FPU problem. If the energy in the FPU chain is reduced beyond the values originally used by FPU, recurrence will become partial, with less and less energy flowing back and forth. In the limit of zero energy, the fraction of energy flowing tends to zero. Therefore the fact that recurrence is not observed in the turbulence case, is not surprising as such. It could be that there is partial recurrence and that this recurrence becomes more visible with increasing energy (of course before the equipartition limit is reached). In our future work we will make a detailed investigation of this point.

In our calculations we have assumed the fluid to be inviscid and incompressible, and we have applied periodic boundary conditions. Therefore, we make some general remarks about the possible impact of viscosity, compressibility, and different boundary conditions.

The addition of viscosity alters the problem completely. Without viscosity the kinetic is redistributed over wave number space, while the total amount of energy remains constant. With viscosity the turbulent energy is not conserved anymore. The energy is drained from the spectrum, predominately at the small scales (see, for instance, Tennekes and Lumley ${ }^{15}$ ).

The compressibility introduces another nonlinearity which makes the problem even more complex. Most of the turbulent flows we encounter in real applications are approximately incompressible.

Replacing the periodic boundary conditions by, for instance, Dirichlet or Neumann conditions, will change the problem drastically. The flow close to the boundary will then depend on the distance to that boundary. It is likely that different wavelengths will show different behavior with a significant influence on the turbulence spectrum.

${ }^{1}$ E. Fermi, J. Pasta, and S. Ulam, Los Alamos Report No. LA-1940 (1955); also published in Collected Papers of Enrico Fermi, edited by E. Segre (University of Chicago Press, Chicago, 1965).

${ }^{2}$ S. Flach, M. V. Ivanchenko, O. I. Kanakov, and K. G. Mishagin, Am. J. Phys. 76, 453 (2008).

${ }^{3}$ J. Ford, Phys. Rep. 213, 271 (1992).

${ }^{4}$ J. De Luca, A. J. Lichtenberg, and M. A. Lieberman, Chaos 5, 283 (1995).

${ }^{5}$ D. L. Shepelyansky, Nonlinearity 10, 1331 (1997). 
${ }^{6}$ L. Berchialla, A. Giorgilli, and S. Paleari, Phys. Lett. A 321, 167 (2004). ${ }^{7}$ D. K. Campbell, S. Flach, and Yu. S. Kivshar, Phys. Today 57 (1), 43 (2004).

${ }^{8}$ S. Flach, M. V. Ivanchenko, and O. I. Kanakov, Phys. Rev. E 73, 036618 (2006).

${ }^{9}$ D. K. Campbell, P. Rosenau, and G. M. Zaslavsky, Chaos 15, 015101 (2005).

${ }^{10}$ G. Gallavotti, Lecture Notes in Physics, No. 728: The Fermi-Pasta-Ulam Problem: A Status Report (Springer, Berlin, 2007).
${ }^{11}$ C. W. Gear, Numerical Initial Value Problems in Ordinary Differential Equations (Prentice-Hall, Englewood Cliffs, NJ, 1971).

${ }^{12}$ G. I. Taylor, Proc. R. Soc. London, Ser. A 151, 421 (1935).

${ }^{13}$ R. H. Kraichnan, J. Fluid Mech. 59, 745 (1973).

${ }^{14}$ W. Polifke, "Aspects of helicity in turbulent flow," Ph.D. thesis, City University of New York, 1990.

${ }^{15} \mathrm{H}$. Tennekes and J. L. Lumley, A First Course on Turbulence (MIT Press, Cambridge, 1972). 